\title{
Conductivity and Hall effect characterization of highly resistive molecular-beam epitaxial GaN layers
}

\author{
P. Kordoša) \\ Institute of Thin Film and Ion Technology, Research Centre Jülich, D-52425 Jülich, Germany \\ M. Morvic and J. Betko \\ Institute of Electrical Engineering, Slovak Academy of Sciences, SK-84239 Bratislava, Slovak Republic
}

J. M. Van Hove, A. M. Wowchak, and P. P. Chow

SVT Associates, Eden Prairie, Minnesota 55344

(Received 30 June 2000; accepted for publication 30 August 2000)

\begin{abstract}
Highly resistive molecular beam epitaxial GaN layers are characterized by temperature dependent conductivity and Hall effect measurements. Seven $n$-type GaN samples with room temperature layer resistivity ranging between 8 and $4.2 \times 10^{6} \Omega \mathrm{cm}$ are used in this study. The experimental data are analyzed by considering various transport models such as band and hopping conduction, scattering on charged dislocations and grain boundaries controlled transport. The same defect level of $0.23 \mathrm{eV}$, attributed to nitrogen vacancy, is found for layers with $\rho_{300} \leqslant 3.7 \times 10^{3} \Omega \mathrm{cm}$. The Hall mobility for two lower resistivity layers is influenced mainly by phonon scattering $\left(\mu_{H} \sim T^{x}, x=-1.4\right)$. However, higher resistivity layers show positive mobility power, $x=0.5-0.9$, which can be explained by dominating scattering on charged dislocations. Properties of layers with the highest resistivity $\left(1 \times 10^{5}\right.$ and $\left.4.2 \times 10^{6} \Omega \mathrm{cm}\right)$ and extremely low Hall mobility $\left(6\right.$ and $\left.<0.1 \mathrm{~cm}^{2} \mathrm{~V}^{-1} \mathrm{~s}^{-1}\right)$ are consistent with grain boundary controlled transport. The barrier height between grains of 0.11 $\mathrm{eV}$ and an average grain size of $200 \mathrm{~nm}$ are found. Neither nearest-neighbor or variable range single phonon hopping nor multiphonon hopping can be clearly attributed to the conduction of the layers investigated. (C) 2000 American Institute of Physics. [S0021-8979(00)03923-2]
\end{abstract}

\section{INTRODUCTION}

Intentionally undoped GaN layers usually show $n$-type conductivity with carrier concentration in the $10^{16} \mathrm{~cm}^{-3}$ range and mobility of about $800 \mathrm{~cm}^{2} \mathrm{~V}^{-1} \mathrm{~s}^{-1}$, i.e., the resistivity of such layers is $\leqslant 1 \Omega \mathrm{cm}$. However, highly resistive buffer or isolation layers are needed for various device applications. Unfortunately, little is known about their preparation and conduction mechanisms. Layers with resistivities of $14,3 \times 10^{3}$ and $10^{6} \Omega \mathrm{cm}$ were prepared by controlling the stoichiometry during molecular beam epitaxial (MBE) growth. ${ }^{1,2}$ Another possible way to obtain highly resistive $\mathrm{GaN}$ layers is by acceptor compensation. MBE grown C-doped layers ${ }^{3}$ and vapor phase epitaxial $\mathrm{Zn}$-doped layers ${ }^{4}$ with resistivities of $10^{6}$ and $10^{12} \Omega \mathrm{cm}$, respectively, have been reported. Ion implantation can be also employed for creation of highly resistive $\mathrm{GaN}$ layers, as was reported recently. ${ }^{5-7}$

The temperature dependent carrier concentration and mobility data on intentionally undoped $\mathrm{GaN}$ layers can be described well by band conduction due to shallow single donor states and by considering phonons and ionized impurities as the main scattering centers. It is believed that nitrogen vacancies act as shallow donors, but this is still controversial. ${ }^{8}$ However, temperature dependent Hall effect data on layers with resistivity higher than $14 \Omega \mathrm{cm}$ are not reported in the literature, because conductive substrates were

${ }^{a)}$ Electronic mail: p.kordos@fz.juelich.de used, ${ }^{4}$ or because the Hall coefficient was unmeasurable. ${ }^{1,2,9}$ Hopping conduction with extremely low Hall mobility $\mu_{H}$ $<1 \mathrm{~cm}^{2} \mathrm{~V}^{-1} \mathrm{~s}^{-1}$ is assumed to be responsible for the latter. ${ }^{1,2,10}$ When the concentrations of the autodoping centers and the deep defects are comparable, the layer becomes highly resistive and conduction by hopping among deep centers might occur. It has been reported that the temperature dependent resistivity data are most consistent with multiphonon hopping, rather than nearest-neighbor or variable range single phonon hopping. However many questions concerning hopping in GaN are still unanswered. ${ }^{1,2,11}$ Additionally, models taking into account scattering on charged dislocations resulting in lowering the mobility have been presented. ${ }^{12-14}$ Lower instead of higher mobility at lower doping concentration, widely reported for low doped $n$-GaN, can be explained by the predominant influence of dislocation scattering in these layers. ${ }^{14}$ Recently, the importance of dislocation scattering on electron transport in InGaN/GaN multiple quantum wells ${ }^{15}$ and $\mathrm{AlGaN} / \mathrm{GaN}$ two-dimensional electron gases ${ }^{16}$ has been also reported. Finally, a model based on potential barriers at grain boundaries was proposed as being responsible for the unusual behavior of highly resistive GaN layers. ${ }^{17,18}$ This model considers that free carriers can be trapped at grain boundaries, resulting in reducing the carrier mobility and thus enhancing the layer resistivity.

In this article, we report on the transport properties of highly resistive GaN layers grown by MBE to extend knowledge of carrier transport behavior in GaN. Seven $n$-type GaN layers with room temperature resistivities ranging between 8 
TABLE I. Layer thickness and $300 \mathrm{~K}$ values of resistivity, Hall concentration and Hall mobility for GaN samples.

\begin{tabular}{ccccc}
\hline \hline $\begin{array}{c}\text { Sample } \\
\text { No. }\end{array}$ & $\begin{array}{c}d \\
(\mu \mathrm{m})\end{array}$ & $\begin{array}{c}\rho_{300} \\
(\Omega \mathrm{cm})\end{array}$ & $\begin{array}{c}n_{H, 300} \\
\left(\mathrm{~cm}^{-3}\right)\end{array}$ & $\begin{array}{c}\mu_{H, 300} \\
\left(\mathrm{~cm}^{2} \mathrm{~V}^{-1} \mathrm{~s}^{-1}\right)\end{array}$ \\
\hline 1 & 3.5 & 8 & $2.5 \times 10^{15}$ & 262 \\
2 & 2.2 & 32 & $6 \times 10^{14}$ & 305 \\
3 & 1 & $2 \times 10^{3}$ & $1.5 \times 10^{14}$ & 30 \\
4 & 2 & $2.3 \times 10^{3}$ & $7 \times 10^{13}$ & 32 \\
5 & 2 & $3.7 \times 10^{3}$ & $8 \times 10^{13}$ & 22 \\
6 & 1.9 & $1 \times 10^{5}$ & $1 \times 10^{13}$ & 6 \\
7 & 0.5 & $4.2 \times 10^{6}$ & $<5 \times 10^{12}$ & $<0.1$ \\
\hline \hline
\end{tabular}

and $4 \times 10^{6} \Omega \mathrm{cm}$ are characterized by temperature dependent conductivity and Hall effect measurements. The experimental data are analyzed by considering the various transport models mentioned above.

\section{EXPERIMENT}

The undoped GaN layers were grown on (0001) basal plane, 2 in. sapphire substrates by MBE using a water cooled radio frequency atomic nitrogen plasma source (SVT Associates rf 4.5) ${ }^{19}$ Conventional effusion cells were used to provide the $\mathrm{Ga}$ and $\mathrm{Al}$ fluxes. Low temperature AlN buffers of different thicknesses were grown after nitridation of the sapphire. The substrate temperature was then increased to $750{ }^{\circ} \mathrm{C}$ for $\mathrm{GaN}$ growth which was done under slightly Garich flux ratios. The layers are about $2 \mu \mathrm{m}$ thick (see Table I) and their crystallinity is nearly the same; the (0002) x-ray rocking curve full width half maximum is $\cong 7$ arcmin. Some samples were characterized by scanning electron microscopy (SEM) and atomic force microscopy (AFM), as well as by low-temperature photoluminescence.

Samples of about $6 \times 6 \mathrm{~mm}^{2}$ were cut from each wafer and In contacts were alloyed at $850^{\circ} \mathrm{C}$. The ohmic behavior of the contacts was confirmed by current-voltage characteristics and lower annealing temperature did not give satisfactory results. Accurate temperature dependent (290-410 K) conductivity and low magnetic field $(0.5 \mathrm{~T})$ Hall effect measurements were carried out in the dark using a highimpedance system. It should be noted that we observed a slight difference in the measured data if the temperature ramp was increased or decreased (about 30\% at room temperature and decreased tendency with increased temperature). This effect can be connected to persistent photoconductivity. ${ }^{20}$ All measurements reported here were performed by using a decreasing temperature ramp, which produces more reasonable results.

\section{RESULTS AND DISCUSSION}

\section{A. Conductivity and Hall effect measurements}

The room temperature $(300 \mathrm{~K})$ values of resistivity, apparent Hall concentration and apparent Hall mobility are summarized in Table I for the GaN samples investigated. The resistivity ranges between 8 and $4.2 \times 10^{6} \Omega \mathrm{cm}$ and the Hall mobility decreases from 305 to $<0.1 \mathrm{~cm}^{2} \mathrm{~V}^{-1} \mathrm{~s}^{-1}$ with increasing resistivity. Note that on sample No. 7 , the one

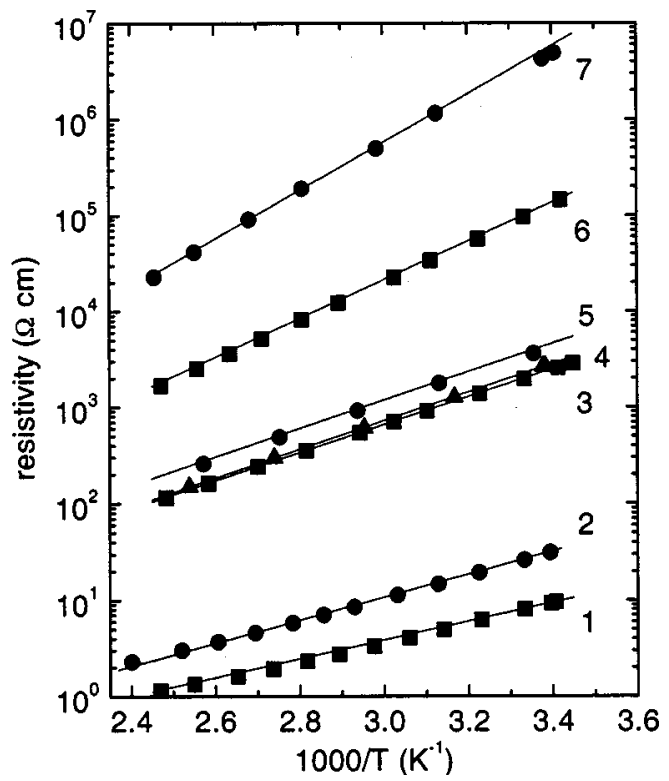

FIG. 1. Temperature dependence of resistivity for GaN layers. The solid lines are theoretical fits according to Eq. (1).

with the highest resistivity, it was not possible to measure the Hall effect at temperatures around $300 \mathrm{~K}$ satisfactorily.

Figures 1 and 2 show the resistivity and apparent (i.e., measured) Hall concentration as a function of inverse temperature for $\mathrm{GaN}$ samples investigated in this study. Resistivity, a more convenient quantity by which to characterize a material property, is shown in Fig. 1 instead of conductivity. The experimental data for both conductivity and Hall concentration show that thermally activated carriers dominate, $\sigma$ $(=1 / \rho)$ and $n_{H}$ are proportional to $\exp (-E / k T)$, where $E$ is the activation energy. Note that the carriers are electrons deduced from the negative sign of the Hall coefficient.

Temperature dependence of the apparent Hall mobility (on a $\log -\log$ scale) for $\mathrm{GaN}$ layers is shown in Fig. 3. The

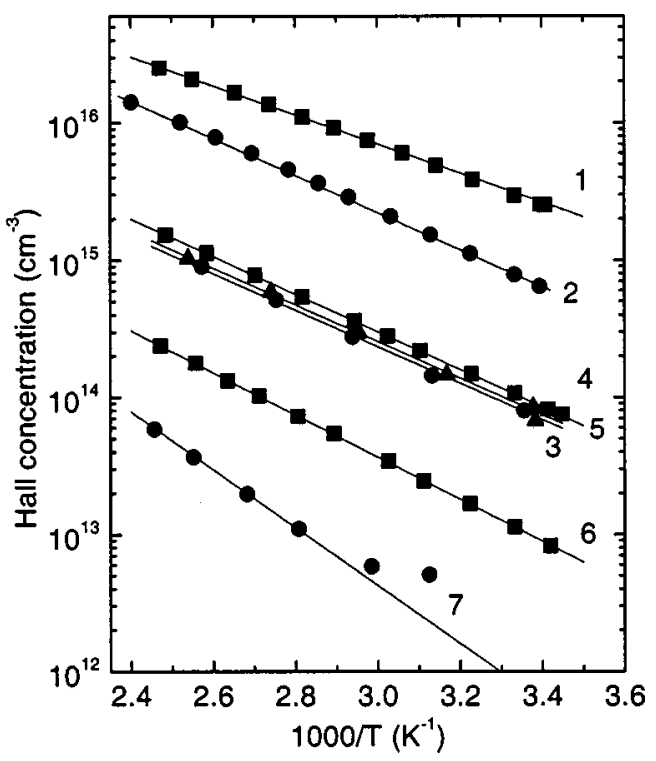

FIG. 2. Temperature dependence of apparent Hall concentration for GaN layers. The solid lines are theoretical fits according to Eq. (10). 


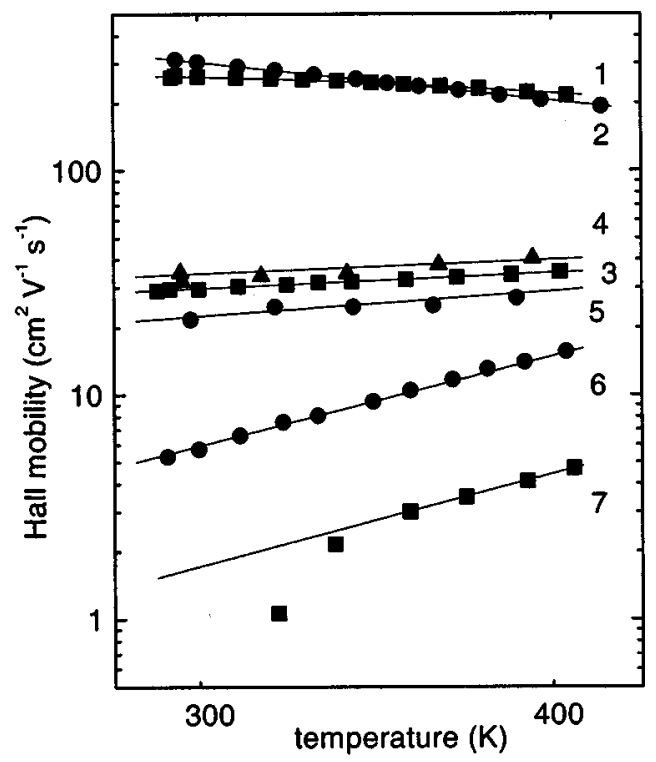

FIG. 3. Temperature dependence of apparent Hall mobility (log-log scale) for GaN layers. The solid lines are theoretical fits according to Eq. (3).

Hall mobility of lower resistive layers 1 and 2 decreases with increasing temperature. On the other hand, for all other layers an increase of $\mu_{H}$ vs $T$ exists, which is more pronounced for layers 6 and 7, those with the highest resistivity and the lowest mobility.

\section{B. Band conduction}

The experimental temperature dependencies of the resistivity, apparent Hall concentration and Hall mobility, shown in Figs. 1-3, can be fitted well by

$$
\begin{aligned}
& \rho=1 / \sigma=C_{\rho} \exp \left(E_{\rho} / k T\right), \\
& n_{H}=C_{n} T^{3 / 2} \exp \left(-E_{n} / k T\right),
\end{aligned}
$$

and

$$
\mu_{H}=C_{\mu} T^{x}
$$

where $C_{\rho}, C_{n}$ and $C_{\mu}$ are temperature independent constants, $E_{\rho}$ and $E_{n}$ are the activation energies and $x$ is the Hall mobility scattering power.

Activation energies evaluated from the $\rho$ vs $1 / T$ dependencies (Fig. 1), commonly used in the literature, as well as from the $n_{H} T^{3 / 2}$ vs $1 / T$ dependencies (not shown here) are summarized in Table II. Both activation energies $E_{\rho}$

TABLE II. Activation energies $E_{\rho}$ and $E_{n}$ and the Hall mobility power $x$ for GaN layers.

\begin{tabular}{ccccc}
\hline $\begin{array}{c}\text { Sample } \\
\text { No. }\end{array}$ & $\begin{array}{c}\rho_{300} \\
(\Omega \mathrm{cm})\end{array}$ & $\begin{array}{c}E_{\rho} \\
(\mathrm{eV})\end{array}$ & $\begin{array}{c}E_{n} \\
(\mathrm{eV})\end{array}$ & $x$ \\
\hline 1 & 8 & 0.20 & 0.17 & $\ldots$ \\
2 & 32 & 0.24 & 0.23 & -1.4 \\
3 & $2 \times 10^{3}$ & 0.29 & 0.23 & 0.56 \\
4 & $2.3 \times 10^{3}$ & 0.29 & 0.23 & 0.5 \\
5 & $3.7 \times 10^{3}$ & 0.29 & 0.23 & 0.9 \\
6 & $1 \times 10^{5}$ & 0.41 & 0.26 & 3.2 \\
7 & $4.2 \times 10^{6}$ & 0.50 & 0.36 & 3.3 \\
\hline \hline
\end{tabular}

$=0.19-0.50 \mathrm{eV}$ and $E_{n}=0.17-0.36 \mathrm{eV}$ increase with increasing layer resistivity. Activation energies $E_{\rho}=0.016$, 0.11 and $0.38 \mathrm{eV}$ were found before on highly resistive GaN with $\rho_{300}=14,3.1 \times 10^{3}$ and $1.9 \times 10^{6} \Omega \mathrm{cm}$, respectively. ${ }^{1}$ These values are evidently lower than those obtained here for similarly resistive samples. Donor levels in the $0.25-0.45 \mathrm{eV}$ range, evaluated from the thermally activated conductivity and attributed to nitrogen vacancies, have been mentioned before. ${ }^{12}$ The nitrogen vacancies could be responsible for yellow band photoluminescence ${ }^{8}$ which we indeed detected on sample Nos. 1 and 2 (other samples were not analyzed). Recently, the preparation of highly resistive $n$-type GaN layers by implantation with various ions like $\mathrm{Ti}, \mathrm{O}, \mathrm{Fe}$ and $\mathrm{Cr}$ has been reported. Donor-like defect levels of 0.20 and $0.48-0.50 \mathrm{eV}$, evaluated from the $\rho$ vs $1 / T$ dependencies, have been found. ${ }^{6}$ These are also comparable values to our $0.20-0.29$ and $0.41-0.50 \mathrm{eV}$ obtained from sample Nos. $1-5$ and $6-7$, respectively. However, it is questionable if such a broad defect band of $0.20-0.50 \mathrm{eV}$, as supposed in Ref. 6, can exist in $\mathrm{GaN}$. It is known, however, that GaAs grown by $\mathrm{MBE}$ at low temperatures is nonstoichiometric and contains a very high number of native defects $\left(\cong 10^{20} \mathrm{~cm}^{-3}\right)$ believed to be mainly arsenic antisites. Its conduction at room temperature is mainly by hopping among defect centers ${ }^{21}$ and the estimated width of the defect band is about $0.15 \mathrm{eV} .^{22}$

\section{Hopping conduction}

We will discuss the data obtained on samples investigated from the hopping conduction point of view. It was already reported that the conduction mechanism in $\mathrm{GaN}$ with a higher number of defect centers can be determined by hopping among localized defects. ${ }^{1,2,10}$ It was found that the experimental data are most consistent with multiphonon (mp) hopping, rather than nearest-neighbor (nn) or variable range (vr) single phonon hopping. ${ }^{1}$ We have analyzed our data considering all three types of hopping.

Conductivity for nearest-neighbor hopping and variable range hopping can be expressed as follows:

$$
\sigma_{\mathrm{nn}}=C_{\mathrm{nn}} \exp \left(-\epsilon_{\mathrm{nn}} / k T\right),
$$

and

$$
\sigma_{\mathrm{vr}}=C_{\mathrm{vr}} \exp \left[-\left(T_{0} / T\right)^{1 / 4}\right],
$$

where $C_{\mathrm{nn}}$ and $C_{\mathrm{vr}}$ are temperature independent constants, $\epsilon_{\mathrm{nn}} \cong e^{2} N_{D}^{1 / 3} / 4 \pi \epsilon \epsilon_{0}$ and $T_{0} \cong 9 \times 10^{18} N_{D}^{-2 / 3}$.

Multiphonon hopping is a combination of both types of single phonon hopping, ${ }^{23}$ and the conductivity can be expressed as

$$
\sigma_{\mathrm{mp}}=C_{\mathrm{mp}} \exp \left(-\epsilon_{\mathrm{nn}} / k T\right) \exp \left[-\left(T_{0} / T\right)^{1 / 4}\right],
$$

where $C_{\mathrm{mp}}$ is a temperature independent constant.

Although our experimental data can be fitted well assuming nearest-neighbor hopping (solid lines in Fig. 1 and $\epsilon_{\mathrm{nn}}=E_{\rho}$ shown in Table II) the donor concentrations evaluated from $\epsilon_{\mathrm{nn}}$ are unrealistically high, $N_{D}=2 \times 10^{21}-4$ $\times 10^{22} \mathrm{~cm}^{-3}$. This is similar to data reported previously for highly resistive GaN. ${ }^{1}$

As is shown in Fig. 4, our experimental data are in good agreement with variable range single phonon hopping, i.e., 


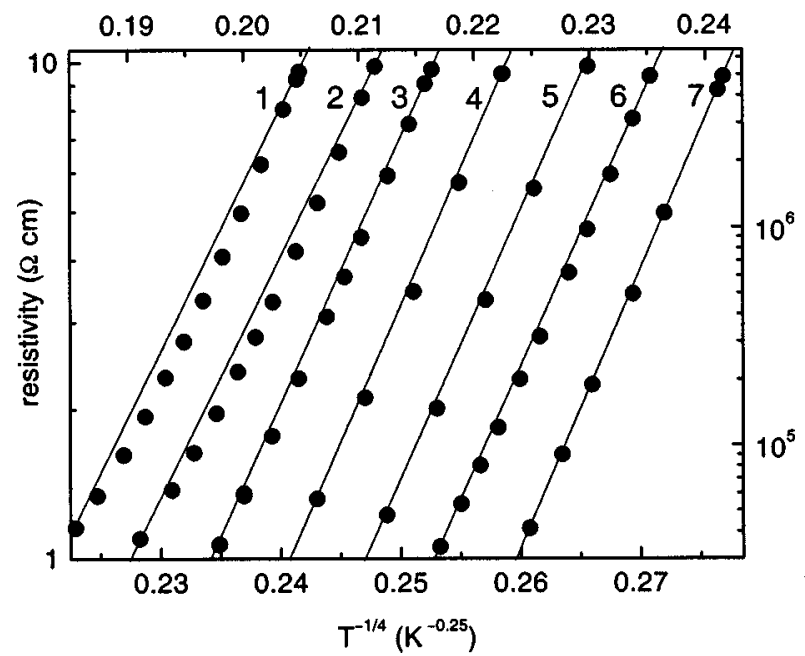

FIG. 4. Resistivity vs $T^{-1 / 4}$ dependence for GaN layers. The solid lines are theoretical fits for single phonon variable range hopping according to Eq. (5). Note that left-bottom axes and right-upper axes are only for sample Nos. 1 and 7, respectively; for other samples the axes have arbitrary units.

with Eq. (5), only for sample No. 7. Unfortunately, the donor concentration of $5 \times 10^{13} \mathrm{~cm}^{-3}$ evaluated from $T_{0}$ is also unrealistic. Fitting of Eq. (6) to our data, i.e., for multiphonon hopping, is shown in Fig. 5. Even though better agreement is obtained for all samples, the resulting $N_{D}$ values obtained from $T_{0}$ and $\epsilon_{\mathrm{nn}}$ are not acceptable. Thus, the question of hopping in GaN needs to be studied in more detail.

\section{Influence of charged dislocations}

The growth of GaN on sapphire substrates is highly lattice mismatched $(\approx 15 \%)$ which leads to a high density of dislocations, typically $10^{8}-10^{10} \mathrm{~cm}^{-2}$. It is assumed that dislocations act as acceptor-like centers which can capture electrons. ${ }^{12-14}$ A space charge region is formed along the dislocation line, resulting in a reduction of carrier mobility.

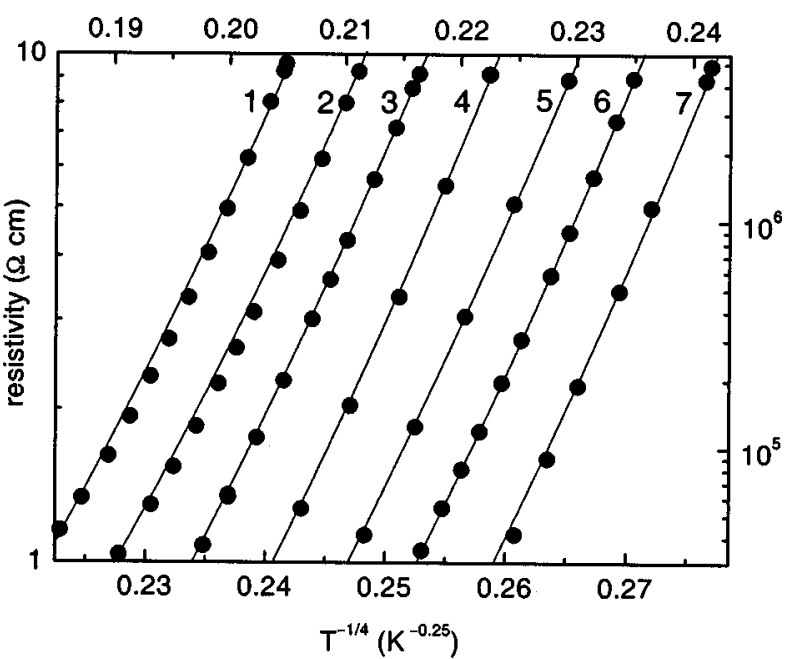

FIG. 5. Resistivity vs $T^{-1 / 4}$ dependence for GaN layers. The solid lines are theoretical fits for multiphonon hopping according to Eq. (6). Note that left-bottom axes and right-upper axes are only for sample Nos. 1 and 7, respectively; for other samples the axes have arbitrary units.
The well known discrepancy that the electron mobility in $\mathrm{GaN}$ decreases with decreasing carrier concentration (below a certain value) has been explained just by influence of a dislocation scattering. ${ }^{13,14}$

According to dislocation scattering theory ${ }^{24}$ the drift mobility depends on temperature as

$$
\mu_{\text {disl }}=C_{d} T^{3 / 2} / \lambda,
$$

where $C_{d}$ is a temperature independent constant and

$$
\lambda=\left(\epsilon \epsilon_{0} k T / e^{2} n_{s}\right)^{1 / 2}
$$

is the screening parameter, where $n_{s}$ is the effective screening concentration.

From Eqs. (7) and (8) one can obtain

$$
\mu_{\text {disl }}=C_{\text {disl }} T^{x},
$$

which is identical in form to Eq. (3). The mobility power $x$ for dislocation scattering will be positive, $x=1$, if the temperature dependence of $n_{s}$ is neglected.

The Hall mobility power $x$, resulting from the fit to experimental $\mu_{H}=f(T)$ data on the samples investigated (Fig. 3 ), is shown in Table II. Lower resistive layers 1 and 2 show a decrease of the Hall mobility with increasing temperature, i.e., $x$ is negative. This indicates that the scattering on charged dislocations does not play a significant role in their conduction in the temperature range used. For sample Nos. 3,4 and 5 the mobility power $x$ is $0.56,0.5$ and 0.9 , respectively. Thus, the carrier transport in these three samples should be mainly influenced by interaction with dislocations, in contradiction to sample Nos. 1 and 2. However, mobility power of 3.2 and 3.3 is found for sample Nos. 6 and 7, respectively. From this it follows that the conduction in these two highest resistivity samples should be influenced by a property other than dislocations. We tried to fit the theoretical dependencies to the experimental data shown in Fig. 3 by considering combined scattering on phonons ( $\mu_{\mathrm{ph}}$ $=C_{\mathrm{ph}} T^{-3 / 2}$ ) and charged dislocations [Eq. (9) with $x=1$ ] using Matthiessen's rule $\mu_{\text {total }}^{-1}=\Sigma \mu_{i}^{-1}$. For sample Nos. 1-5 we obtained very good fits (the results are practically identical to the solid lines shown in Fig. 3). Poor fits resulted from using this procedure on sample Nos. 6 and 7 as expected.

\section{E. Potential barriers at grain boundaries}

Besides a very high number of structural defects due to the high lattice mismatch between $\mathrm{GaN}$ and the sapphire substrate, polycrystalline growth can also occur. ${ }^{25}$ In such a case the transport properties are strongly influenced by boundaries between ordered grains, mainly if lateral transport occurs. Potential barriers exist at grain boundaries which act as traps of free carriers. According to transport theory in polycrystalline layers ${ }^{26}$ both the carrier concentration $n$ and the mobility $\mu$ are thermally activated, i.e.,

$$
n=n_{0} \exp \left(-E_{n b} / k T\right),
$$

and

$$
\mu=\mu_{0} \exp \left(-\Phi_{b} / k T\right),
$$




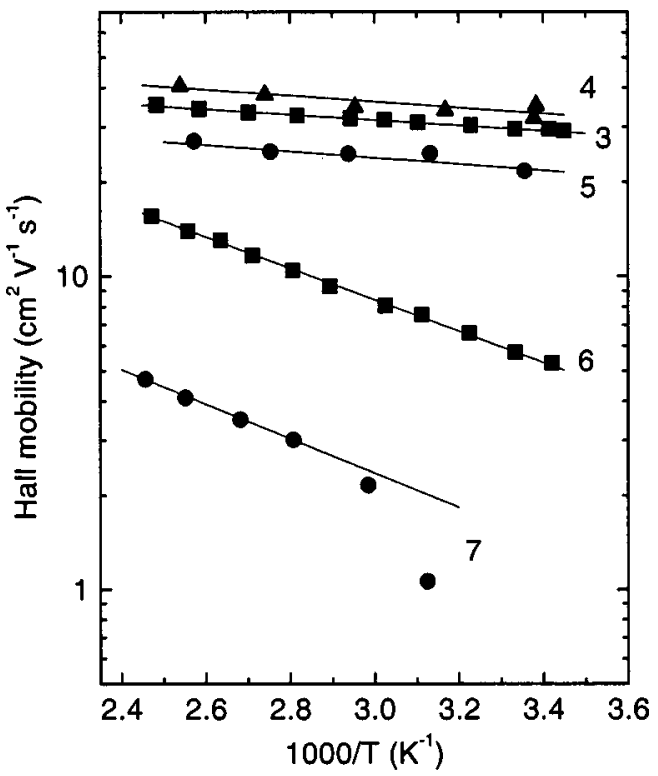

FIG. 6. Apparent Hall mobility as a function of inverse temperature for GaN layers. The solid lines are theoretical fits according Eq. (11).

where $E_{n b}$ is the activation energy, $\Phi_{b}$ is the barrier height at the grain boundaries and $n_{0}$ and $\mu_{0}$ are temperature independent constants.

The unusual optical and electrical properties of GaN grown on sapphire have been explained by the existence of the polycrystalline microstructure in the layers. ${ }^{17}$ Activation energies and barrier heights up to 0.29 and $0.09 \mathrm{eV}$, respectively, have been found in a more detailed study on highly resistive MBE GaN layers by considering grain boundary controlled transport. ${ }^{18}$ Recently this model has been applied to explain the properties of $\mathrm{He}$ implanted GaN layers. ${ }^{7}$ From the conductivity measurements, the energy evaluated $\left(E_{n b}\right.$ $+\Phi_{b}$ ) increases with increased implantation dose (i.e., with decreasing net carrier concentration) and saturates at about $0.8 \mathrm{eV}$. From analysis an average grain size of $150 \mathrm{~nm}$ follows for the samples used. However, experimental evidence of a polycrystalline microstructure was not given in these studies. ${ }^{7,18}$

Fitting of Eqs. (10) and (11) to our experimental data is shown in Figs. 2 and 6 (solid lines), respectively. Note that the mobility data for sample Nos. 1 and 2 are not shown in Fig. 6 because their analysis is irrelevant. The resulting activation energies are $E_{n b}=0.27,0.30$ and $0.41 \mathrm{eV}$ for sample Nos. 3-5, 6 and 7, respectively. For the barrier height at grain boundaries we obtained $\Phi_{b}=0.017-0.020,0.10$ and $0.11 \mathrm{eV}$ for sample Nos. 3-5, 6 and 7, respectively. All values are in good agreement with those published previously on highly resistive $\mathrm{GaN}^{7,18}$ In order to verify if it is reasonable to apply this analysis to our samples we investigated their surface microstructure by SEM and AFM techniques. Sample Nos. 1 and 2 show relatively smooth surfaces without any remarkable macroscopic defects [see Fig. 7(a)]. The surface of sample Nos. 3-5 is rough, but still compact. However, for sample No. 7, i.e., the one with the highest resistivity of $4.2 \times 10^{6} \Omega \mathrm{cm}$, we found a polycrystalline structure. The SEM micrograph shown in Fig. 7(b) docu-

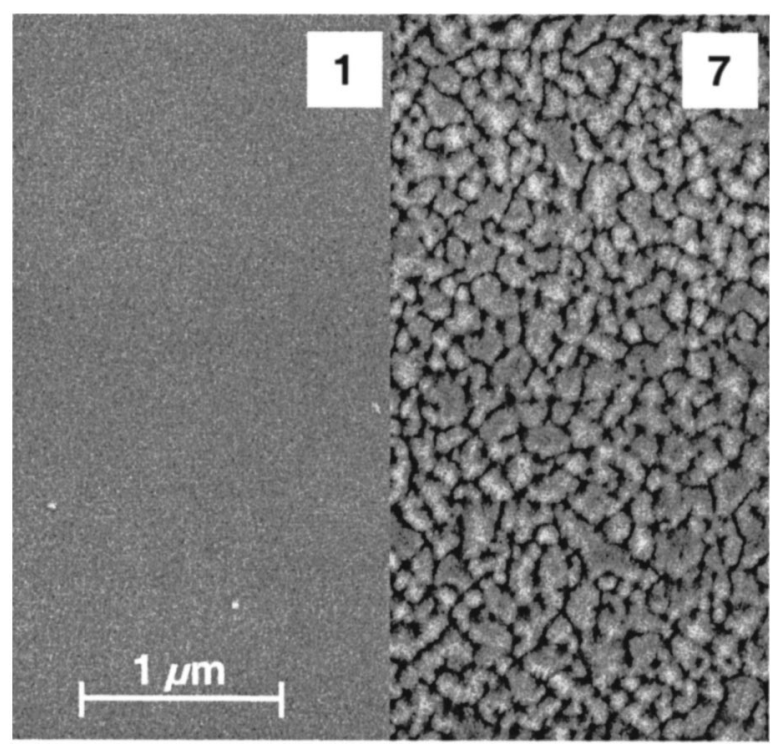

FIG. 7. SEM micrographs of the GaN surface: (a) layer 1 with resistivity of $8 \Omega \mathrm{cm}$ and (b) layer 7 with resistivity of $4.2 \times 10^{6} \Omega \mathrm{cm}$.

ments it. An average grain size of about $200 \mathrm{~nm}$ is estimated from both SEM and AFM micrographs. Sample No. 6 should also exhibit a polycrystalline microstructure, as follows from transport analysis. Unfortunately, we have not found such a surface property. This can be connected to the different layer thickness of these two samples $(1.9 \mu \mathrm{m}$ for sample No. 6, but only $0.5 \mu \mathrm{m}$ for sample No. 7), i.e., in the case of the thicker sample the polycrystalline region might be overgrown.

\section{CONCLUSIONS}

We performed temperature dependent conductivity and Hall effect measurements on highly resistive GaN layers grown by MBE. The experimental data were analyzed by considering various transport models like band and hopping conduction, scattering on charged dislocations and grain boundary controlled transport. The main results of our investigation may be summarized as follows.

(i) Transport properties of layers which exhibit room temperature resistivity of 8 and $32 \Omega \mathrm{cm}$ and Hall mobility of 262 and $305 \mathrm{~cm}^{2} \mathrm{~V}^{-1} \mathrm{~s}^{-1}$ can be described well by band conduction. A defect level of $0.23 \mathrm{eV}$ is found which is attributed to a nitrogen vacancy. The Hall mobility is influenced mainly by phonon scattering.

(ii) On layers with $\rho=(2-3.7) \times 10^{3} \Omega \mathrm{cm}$ and $\mu_{H}$ $=22-32 \mathrm{~cm}^{2} \mathrm{~V}^{-1} \mathrm{~s}^{-1}$ the same defect level of $0.23 \mathrm{eV}$ is observed. However, $\mu_{H} \sim T^{x}$ dependence with mobility power $x=0.5-0.9$ is observed, which can be explained by dominating scattering on charged dislocations.

(iii) Properties of layers with the highest resistivity (1 $\times 10^{5}$ and $4.2 \times 10^{6} \Omega \mathrm{cm}$ ) and extremely low Hall mobility ( 6 and $<0.1 \mathrm{~cm}^{2} \mathrm{~V}^{-1} \mathrm{~s}^{-1}$ ) are consistent with grain boundary controlled transport. This is confirmed by the polycrystalline microstructure which has an average grain size of about 200 $\mathrm{nm}$, found by SEM and AFM investigations.

(iv) From the hopping analysis it follows that neither nearest-neighbor or variable range single phonon hopping 
nor multiphonon hopping can be clearly attributed to the conduction of the highly resistive GaN samples investigated.

\section{ACKNOWLEDGMENTS}

The authors wish to thank P. Javorka for van der Pauw sample preparation, M. Grimm for X-ray diffractometry, J. Skriniarova and P. Bochem for SEM and AFM micrographs and R. Schmidt for photoluminescence measurements.

${ }^{1}$ D. C. Look, D. C. Reynolds, W. Kim, O. Aktas, A. Botcharev, A. Salvador, and H. Morkoç, J. Appl. Phys. 80, 2960 (1996).

${ }^{2}$ D. C. Look, Mater. Sci. Eng., B 50, 50 (1997).

${ }^{3}$ J. B. Webb, H. Tang, S. Rolfe, and J. A. Bardwell, Appl. Phys. Lett. 75, 953 (1999).

${ }^{4}$ N. I. Kuznetsov, A. E. Nikolaev, A. S. Zubrilov, Yu. V. Melnik, and V. A. Dimitriev, Appl. Phys. Lett. 75, 3138 (1999).

${ }^{5}$ C. Uzan-Saguy, J. Salzman, R. Kalish, V. Richter, U. Tish, and S. Zamir, Appl. Phys. Lett. 74, 2441 (1999).

${ }^{6}$ X. A. Cao, S. J. Pearton, G. T. Dang, A. P. Zhang, F. Ren, R. G. Wilson, and J. M. Van Hove, J. Appl. Phys. 87, 1091 (2000).

${ }^{7}$ J. Salzman, C. Uzan-Saguy, R. Kalish, V. Richter, and B. Meyler, Appl. Phys. Lett. 76, 1431 (2000).

${ }^{8}$ S. C. Jain, M. Willander, J. Narayan, and R. Van Overstraeten, J. Appl. Phys. 87, 965 (2000).

${ }^{9}$ Ch. Wang and R. F. Davis, Appl. Phys. Lett. 63, 990 (1993).
${ }^{10}$ R. J. Molnar, T. Lei, and T. D. Moustakas, Appl. Phys. Lett. 62, 72 (1993).

${ }^{11}$ P. Kordoš, P. Javorka, M. Morvic, J. Betko, J. M. Van Hove, A. M. Wowchak, and P. P. Chow, Appl. Phys. Lett. 76, 3762 (2000).

${ }^{12}$ T. L. Tansley and R. J. Egan, Physica B 185, 190 (1993).

${ }^{13}$ H. M. Ng, D. Doppalapudi, T. D. Moustakas, N. G. Weimann, and L. F. Eastman, Appl. Phys. Lett. 73, 821 (1998).

${ }^{14}$ D. C. Look and J. R. Sizelove, Phys. Rev. Lett. 82, 1237 (1999).

${ }^{15}$ I. Lo, K. Y. Hsieh, S. L. Hwang, L. W. Tu, W. C. Mitchel, and A. W. Saxler, Appl. Phys. Lett. 74, 2167 (1999).

${ }^{16}$ D. Jena, A. C. Gossard, and U. K. Mishra, Appl. Phys. Lett. 76, 1707 (2000).

${ }^{17}$ S. D. Hersee, J. C. Ramer, and K. J. Malloy, MRS Bull. July, 45 (1997).

${ }^{18}$ M. Fehrer, S. Einfeldt, U. Birkle, T. Gollnik, and D. Hommel, J. Cryst. Growth 189/190, 763 (1998).

${ }^{19}$ J. M. Van Hove, G. J. Cosimini, E. Nelson, A. M. Wowchak, and P. P. Chow, J. Cryst. Growth 150, 908 (1995).

${ }^{20}$ C. V. Reddy, K. Balkhrishnan, H. Okumura, and S. Yoshida, Appl. Phys. Lett. 73, 244 (1998)

${ }^{21}$ M. Morvic, J. Betko, J. Novák, A. Förster, and P. Kordǒ̌, Phys. Status Solidi B 205, 125 (1998).

${ }^{22}$ Y. H. Chen, Z. Yang, Z. G. Wang, and R. G. Li, Appl. Phys. Lett. 72, 1866 (1998)

${ }^{23}$ D. Emin, Phys. Rev. Lett. 32, 303 (1974).

${ }^{24}$ B. Pödör, Phys. Status Solidi 16, K167 (1966).

${ }^{25}$ O. Ambacher, J. Phys. D 31, 2653 (1998).

${ }^{26}$ J. W. Orton and M. J. Powell, Rep. Prog. Phys. 43, 1263 (1980). 